\section{Cancer cytogenetics: methods and protocols}

Edited by J Swansbury. Totowa, NJ: Humana Press, 2003, £70 (US\$89.50), pp 296. ISBN 1588290808

The value of cytogenetic studies for diagnosis and prognosis in malignancy is well established and this book sets out to provide the information required to set up a successful diagnostic laboratory in this challenging branch of cytogenetics. Unlike the other volumes in the Methods in Molecular Biology $^{\mathrm{TM}}$ series, the emphasis in this book is on the establishment of a complete diagnostic service rather than simply listing methodologies and protocols for the technical aspects of this work. The book includes advice on the training of staff, the interpretation of findings, and the appropriate focus for limited resources in view of the rapidly increasing demand for diagnostic cytogenetic studies in malignancy.

The format of the book is very straightforward with a chapter on the background relating to a disease, for example myeloid disorders, followed by a chapter on the cytogenetic techniques required for the investigation of that disease. Following the introductory chapter, all of the common haematological malignancies are covered in the first eight chapters. These are followed with two chapters on the background and technical aspects of solid tumour cytogenetics. The next chapter provides some excellent advice on the analysis and interpretation of cytogenetic findings in malignancy. The following section of four chapters covers the emerging fluorescence in situ hybridisation (FISH) technologies and their application to haematological cytogenetics. These are followed by a very interesting chapter discussing the difficult choices to be made when trying to balance the differing requirements of research and diagnostic work and funding of new technologies. The final chapter provides valuable basic guidance on chromosome recognition, the most fundamental skill in cytogenetics. Curiously, though, this chapter does not include a picture of a normal karyotype. This book does not cover the complementary molecular techniques involved in malignancy genetics and the molecular cytogenetic aspects of leucaemia cytogenetics are not as well covered as the routine chromosome analysis. For example, there is a chapter on FISH for acute lymphoblastic disorders, but no chapter on the application of FISH in myeloid disorders. However, this book concentrates more on the options available and the problems associated with FISH in the laboratory, and the technical aspects are more comprehensively covered in the companion volume, Molecular cytogenetics: protocols and applications, which is volume 204 in the Methods in Molecular Biology $^{\mathrm{TM}}$ series. A minor irritation I found with this book was in the proof reading (or lack of it). As well as minor typographical errors, I found a repeated sentence and on one page, whole chromosome paints referred to alternately as "WCPs" and "WPCs". This could cause confusion to the reader and in a book describing technical procedures, errors such as a misplaced decimal point may have significant consequences.

There are 10 renowned contributors to this volume. However, 11 of the 18 chapters, including all the background chapters, have been written by the editor, John Swansbury. I think with his wealth of experience, thoughtful approach, and pleasant writing style this adds to the value of this book, which reads well. Although this book is more appropriate for individuals or laboratories starting out in this field of malignancy cytogenetics, with its up to date references and valuable advice, it deserves a place on the bookshelf of all cancer cytogenetic laboratories.

L Willatt

\section{Cleft lip and palate. From origin to treatment}

Edited by Diego Wyszynski. Oxford: Oxford University Press, 2002, £110, pp 518. ISBN 0195139062

This compendium of 42 chapters purports to provide graduate students, practitioners, researchers, parents, and siblings with information that can facilitate efforts to reduce rates of cleft lip and palate and to increase the quality and years of healthy life of individuals who are born with these conditions. It succeeds admirably. It is difficult to put together such a comprehensive volume that covers issues from very basic embryology and genetics through to family based resources and support groups. Dr Wyszynski has assembled an impressive collection of experts in the field and has succeeded in providing individual chapters that are comprehensive and up to date in their coverage and reasonably well integrated as well. Although some subjects are covered in more depth than others, there appear to be no major omissions from the overall effort and in several cases chapters are the best summaries as well as the most detailed overviews on the topic that have yet been written. Many individual chapters will be able to serve as reference points for years to come. Several chapters have been particularly effective in providing detailed bibliographies as well as summary tables.

In any summary this large, one could certainly find weaknesses to nitpick. Some chapters overlap and fail to be integrated.
Others do not provide the depth of information or background that one might desire, and the glossary and index are relatively short for the amount of information contained in the volume as a whole and appear to overemphasise genetic and embryologic findings and underemphasise clinical and patient care aspects. Some individual hot topics such as distraction osteogenesis were difficult to find mention of at all, while others were covered repeatedly in several chapters, sometimes with conflicting interpretations.

These minor criticisms aside, the volume has much to recommend it. Numerous tables provide comprehensive summaries of epidemiological, genetic, embryological, surgical approaches, and incidence/prevalence data. It is well illustrated with embryological and surgical views and interpretations and provides a number of in depth discussions of controversial points including clinical trials in surgery, recurrence prevention strategies, and epidemiological approaches. Some particular strengths also include an emphasis on patient and family based care as well as the availability of resources for researchers, clinicians, and family members.

Although some of the text is inevitably focused on an individual's particular expertise or background, there is overall a strong and successful attempt to integrate international experts with international questions and approaches. Given a disorder such as cleft lip and palate that has such variable distribution around the world and where the availability of care is remarkably inconsistent, this edited text has been very successful in raising important questions that are currently relevant in highly developed countries, including those both with and without national health care systems, as well as in issues that are of compelling importance for less developed countries where the availability of even single contact reconstructive surgery may be difficult for much of the affected population.

I particularly enjoyed reading the clinical and patient care oriented summaries and found much of the tabular summaries very useful and very well done. Because the text is so broad in its task and has been so competently done, it should serve as an invaluable resource for anyone working with cleft lip and palate, whether they be researchers or clinicians. It is a text that truly belongs as a standard reference in not only every clinic that takes care of such patients, but also on the desks of epidemiologists, embryologists, geneticists, nutritionists, speech pathologists, dentists, psychologists, and others who care for such children. It seems likely that this will serve as a classic text for many years to come and one hopes that in the future newly edited and updated versions will become available as well.

J Murray 